\title{
First-pass perfusion CMR two days after infarction predicts severity of functional impairment six weeks later in the rat heart
}

\author{
Daniel J Stuckey ${ }^{1,2^{*}}$, Carolyn A Carr ${ }^{1}$, Stephanie J Meader ${ }^{1}$, Damian J Tyler $^{1}$, Mark A Cole ${ }^{1}$ and Kieran Clarke ${ }^{1}$
}

\begin{abstract}
Background: In humans, dynamic contrast CMR of the first pass of a bolus infusion of Gadolinium-based contrast agent has become a standard technique to identify under-perfused regions of the heart and can accurately demonstrate the severity of myocardial infarction. Despite the clinical importance of this method, it has rarely been applied in small animal models of cardiac disease. In order to identify perfusion delays in the infarcted rat heart, here we present a method in which a $T_{1}$ weighted MR image has been acquired during each cardiac cycle.

Methods and results: In isolated perfused rat hearts, contrast agent infusion gave uniform signal enhancement throughout the myocardium. Occlusion of the left anterior descending coronary artery significantly reduced the rate of signal enhancement in anterior regions of the heart, demonstrating that the first-pass method was sensitive to perfusion deficits. In vivo measurements of myocardial morphology, function, perfusion and viability were made at 2 and 8 days after infarction. Morphology and function were further assessed using cine-MRI at 42 days. The perfusion delay was larger in rat hearts that went on to develop greater functional impairment, demonstrating that first-pass CMR can be used as an early indicator of infarct severity. First-pass CMR at 2 and 8 days following infarction better predicted outcome than cardiac ejection fraction, end diastolic volume or end systolic volume.
\end{abstract}

Conclusion: First-pass CMR provides a predictive measure of the severity of myocardial impairment caused by infarction in a rodent model of heart failure.

\section{Background}

Non-invasive, in vivo measurements of cardiac morphology, viability and function have revolutionised our understanding of cardiac disease [1-3]. In combination, echocardiography, angiography, PET, SPECT, CT and CMR can be used to measure wall and valve motion, cavity volumes, aortic and coronary blood flows, tissue perfusion and cardiac metabolism [1-5]. CMR offers excellent soft tissue contrast, coupled with high temporal and spatial resolution, allowing accurate measurement of cavity volumes, wall thickness and ejection fraction. Further, infarct size and transmurality, measured using CMR shortly after systemic infusion of the contrast agent gadolinium-diethylenetriaminepentaacetate (DTPA), strongly correlates with progression to

\footnotetext{
* Correspondence: daniel.stuckey1@imperial.ac.uk

'Department of Physiology, Anatomy and Genetics, University of Oxford,

Parks Road, Oxford, UK

Full list of author information is available at the end of the article
}

heart failure and patient mortality [2,5-8]. MR imaging of the first-pass of a bolus infusion of Gd-DTPA has become a standard clinical method for identifying under-perfused regions of the human heart and accurately predicts the severity of myocardial ischemia [9-14].

Scaling down imaging techniques to study the ever increasing number of small animal disease models has been a formidable challenge [15]. The rat model of myocardial infarction, induced by occlusion of the left anterior descending coronary artery, is widely used and has provided data essential for the development of surgical and pharmacological therapy [16]. However, the left ventricular mass of the rat heart $(\sim 500 \mathrm{mg})$ is approximately three hundred times smaller than the human left ventricle $(\sim 150 \mathrm{~g})$, and contracts 5 to 10 times faster, making it necessary to use high field strengths, smaller voxel sizes and rapid gating strategies.

\section{Biomed Central}


Small animal cine-MRI has identified increased left ventricular mass, end diastolic and end systolic volumes in the infarcted heart during progressive cardiac remodeling [17]. Pharmocologiical and biological therapies have been extensively tested in this model system $[16,18]$. However, owing to the variability of infarct size induced by experimental myocardial infarction, it is useful to have a baseline measure of infarcted heart function prior to administration of the therapeutic agent. Acute changes to left ventricular mass and volume, typically made using echocardiography, are not good predictors of functional outcome, making these frequently used experimental methods for assessing acute infarct severity inaccurate. A more robust early measure of disease severity may be obtained from late gadolinium enhancement (LGE) CMR [19] or perfusion imaging. LGE-CMR identifies tissue damage due to increased contrast agent retention within infarcted areas of caused by cell membrane rupture and increased interstitial space $[3,5]$. These processes occur within hours of infarction, so offer good early predictors of infarct severity. An even earlier indicator of the extent of functional impairment may be offered by perfusion imaging because it is the reduced perfusion that ultimately results in cell necrosis, membrane rupture, reduced contractility, remodeling and heart failure [10]. Myocardial perfusion has been imaged using arterial spin labeling and $\mathrm{T}_{1}$ mapping [20-23], but imaging the first-pass of a Gd-bolus infusion has rarely been used to study small animal models of myocardial infarction [24,25], probably owing to the need for ultra-fast and high-resolution imaging [20].

Here, we have developed a method to identify perfusion deficits in infarcted rat hearts during bolus infusion by acquiring a $T_{1}$ weighted MR image every cardiac cycle, both in the isolated perfused heart and in vivo. Perfusion delay and myocardial viability, morphology and function were measured in vivo at 2 and 8 days after infarction to compare with cardiac function measured in the same animals at 42 days.

\section{Methods}

\section{Experimental design}

For ex vivo MRI studies of isolated, perfused hearts, control female Wistar rat hearts $(\mathrm{n}=12)$ were isolated, perfused and imaged as described below. Hearts were then removed from the magnet, the coronary artery occluded, the heart repositioned and re-imaged $(\mathrm{n}=8)$.

For in vivo MRI studies, baseline cine-MRI was performed prior to surgery. Female Wistar rats $(\mathrm{n}=34)$ underwent myocardial infarction followed by in vivo first-pass imaging, cine- and LGE-CMR at 2 and 8 days. Problems with i.v. infusions, owing to unsuccessful tail vein cannulation, movement of cannulae during positioning of rats within the magnet and blood clots or air bubbles in the drug line, meant that first-pass imaging could not be performed on all 34 rats at each time point ( 2 days, $\mathrm{n}=21 ; 8$ days, $\mathrm{n}=27$; both 2 and 8 days, $\mathrm{n}=14$ ). After 9 days, 7 of the rats that were imaged at 2 and 8 days were sacrificed; hearts were isolated, perfused and imaged ex vivo. The remaining 27 rats (14 and 20 of which had had first-pass imaging at 2 and 8 days respectively) underwent cine-MRI at 42 days. The University of Oxford Animal Ethics Review Committees and the Home Office (London, UK) approved all procedures.

\section{Ex vivo MRI of isolated, perfused hearts}

Hearts were excised from control $(n=12)$ or infarcted $(\mathrm{n}=7)$ rats, arrested in ice-cold Krebs-Henseleit buffer and attached to a cannula by the ascending aorta. Hearts were perfused at $85 \mathrm{mmHg}$ with filtered KrebsHenseleit buffer containing $11 \mathrm{mM}$ glucose and $1.8 \mathrm{mM}$ pyruvate. Buffer was maintained at $37^{\circ} \mathrm{C}$ and $\mathrm{pH} 7.4$, aerated with $95 \% \mathrm{O}_{2} / 5 \% \mathrm{CO}_{2}$ and not re-circulated, to avoid contrast agent accumulation. A balloon connected to a pressure transducer was placed in the left ventricular (LV) cavity, inflated to an end diastolic pressure of $4 \mathrm{mmHg}$ and used to measure cardiac function. The heart was placed in a $20 \mathrm{~mm}$ NMR tube and lowered into the centre of a vertical-bore, 11.7 T MR magnet (Magnex Scientific, Oxon, United Kingdom) with a $20 \mathrm{~mm}{ }^{1} \mathrm{H}$ birdcage coil (Rapid Biomedical, Würzburg, Germany) and a Bruker console (Bruker Medical, Ettlingen, Germany) running Paravision 2.1.1. Perfusion was then switched to a constant flow of $15-20 \mathrm{ml} / \mathrm{min}$, based on each heart's coronary flow at $85 \mathrm{mmHg}$.

First-pass perfusion imaging was performed to cover the entire LV with repeated acquisitions using a $T_{1}$ weighted fast gradient echo sequence that acquired one image every $128 \mathrm{~ms}$ (TE/TR, $0.8 / 2 \mathrm{~ms} ; 60^{\circ}$ pulse; field of view $20 \times 20 \mathrm{~mm}$; slice thickness $1 \mathrm{~mm}$; matrix size $64 \times$ 64 , zero filled to $256 \times 256$ ). It was determined that rapid image acquisition was preferential to greater spatial resolution as this allowed more images to be acquired during the first pass of contrast agent, gave greater signal to noise and reduced motion artifacts. A bolus of $25 \mu \mathrm{l}$ of $0.5 \mathrm{mmol} / \mathrm{ml}$ Gd-DTPA-BMA (Gadodiamine, Omniscan) was rapidly infused after approximately 10 of the 128 images were acquired. First-pass imaging was repeated during multiple bolus infusions to cover the entire LV. Control hearts were then removed from the magnet, the LAD was permanently occluded using a 5-0 silk suture (successful in 8 hearts) and first-pass imaging was repeated during multiple bolus infusions to cover the entire LV. Saturated gradient echo images were also acquired to determine whether LGE could be detected in the infarcted, perfused heart. 


\section{Coronary occlusion model of myocardial infarction}

Rats $(\mathrm{n}=34)$ were anesthetized with $2.5 \%$ isoflurane in $\mathrm{O}_{2}$, intubated, and a thoracotomy was performed. The pericardium was removed and the heart was infarcted by ligation of the left anterior descending coronary artery (LAD), approximately $2 \mathrm{~mm}$ from the origin, using a 5-0 proline suture [26].

\section{In vivo $\mathrm{CMR}$}

Rats were anesthetized with $2.5 \%$ isoflurane in $\mathrm{O}_{2}$ and positioned supine in a purpose-built cradle. ECG electrodes were inserted into the forepaws, a respiration loop was taped across the chest and heart and respiration signals were monitored using a custom-built physiological motion gating device [27]. The cradle was lowered into a vertical-bore, 11.7 T MR system with a 52-mm birdcage coil (Rapid Biomedical, Würzburg, Germany) and a Bruker console running Paravision 2.1.1.

First-pass CMR was performed on a single short axis slice, which encompassed the papillary muscles, using a fast gradient echo sequence that acquired one image per heart beat (TE/TR, 0.8/2 ms; $60^{\circ}$ pulse; field of view $40 \times$ $40 \mathrm{~mm}$; slice thickness $1.5 \mathrm{~mm}$; matrix size $64 \times 64$, zero filled to $256 \times 256$ ). The flip angle was adjusted to null signal in the myocardium before bolus infusion. A bolus of $0.5 \mathrm{mg} / \mathrm{kg} \mathrm{Gd}$ in $200-270 \mu \mathrm{l}$ saline was infused via the tail vein in 2 seconds during image acquisition. This volume, derived from ex vivo data, was ten times greater than that used in ex vivo experiments as in vivo cardiac output (75$100 \mathrm{ml} / \mathrm{min}$ ) was 5 times greater than ex vivo perfusion rate $(15-20 \mathrm{ml} / \mathrm{min})$ and infusion time was 2 seconds in vivo and less than 1 second ex vivo

Cardiac cine-MRI was performed 10 to 25 min after Gd infusion as described [28]. A stack of contiguous $1.5 \mathrm{~mm}$ thick true short-axis ECG and respiration-gated cineFLASH images (TE/TR $1.43 / 4.6 \mathrm{~ms} ; 25^{\circ}$ pulse; field of view $51.2 \times 51.2 \mathrm{~mm}$; matrix size $256 \times 256$; voxel size $200 \times 200 \times 1500 \mu \mathrm{m} ; 25$ to 35 frames per cardiac cycle (mean heart rate $360 \pm 20 \mathrm{bpm}$ ), were acquired to cover the entire left ventricle. Long-axis two-chamber and four-chamber images were also acquired. The relatively high flip angle $\left(25^{\circ}\right)$ of the cine-MRI acquisition allowed LGE-CMR to be assessed from the same stack of cine images, similar to the method recently described and validated by Protti et al [29]. The entire in vivo imaging protocol was performed in approximately 60 minutes.

\section{MRI data analysis}

Image analysis was performed by an operator blinded to the surgical procedure performed on the rats using ImageJ (NIH Image, Bethesda, MD). Left ventricular volumes and ejection fractions were calculated from the stack of cine images as described [18]. To determine the area of akinetic scar tissue, the average endocardial and epicardial length of akinetic tissue in cine images were measured in each slice, summed across all slices and multiplied by slice thickness $(1.5 \mathrm{~mm})$ to give the absolute scar size as described by Nahrendorf et al [17] Perfusion was assessed by analyzing the change in signal intensity during Gd infusion in regions of interest (ROIs) selected in the infarcted anterior myocardium, adjacent to the infarct in the peri-infarcted lateral region and in the remote tissue of the inferior wall of the left ventricle. The relative rate of perfusion delay was measured as the time from inflow of contrast agent to the LV cavity, to $90 \%$ of the maximum signal intensity in the ROI. From ex vivo perfusion measurements, it was possible to measure the rate of contrast inflow and efflux from the gradient of the signal intensity/time curve. The area of reduced perfusion on the stack of $e x$ vivo images was identified as myocardium with signal intensity greater than two standard deviations below that of remote myocardium.

In vivo LGE-MR images were thresholded to two standard deviations above the mean signal intensity from remote tissue. The volume and perimeter (endocardial length of the enhanced region) of the hyperenhanced region was traced manually and expressed as a percentage of total LV volume or perimeter.

\section{Data and statistical analysis}

Results are shown as means \pm standard errors. Differences were considered significant at $P<0.05$ using unpaired Student t-tests or ANOVAs for repeated measures. Dependence between two measures was assessed using Pearson's Correlations.

\section{Results}

First-pass cardiac perfusion imaging in isolated, perfused rat hearts

Repeated imaging of bolus injections of Gd were performed to determine the contrast agent infusion volume and duration that resulted in maximal contrast within perfused control rat hearts. At a buffer flow rate of $15 \mathrm{ml} / \mathrm{min}$, a rapid (approximately 1 second) injection of $25 \mu \mathrm{l}$ of $0.5 \mathrm{mmol} / \mathrm{ml} \mathrm{Gd}$, directly proximal to the aorta, led to uniform enhancement throughout the myocardium (Figure 1a, b), with time to $90 \%$ maximum enhancement similar for inferior, lateral and anterior regions. The rapid clearance of $\mathrm{Gd}$ from the tissue allowed bolus injections to be repeated every $90 \mathrm{sec}-$ onds. Larger bolus infusions $(>50 \mu \mathrm{l})$ resulted in non uniform signal enhancement, and smaller infusion volumes $(10 \mu \mathrm{l})$ did not enhance at all (Figure 1b). It was also possible to measure the gradient of the signal intensity/time curves at the time of Gd inflow. Again, the rate of signal intensity increase was similar for inferior, lateral and anterior regions (Figure 1d). The rate of 


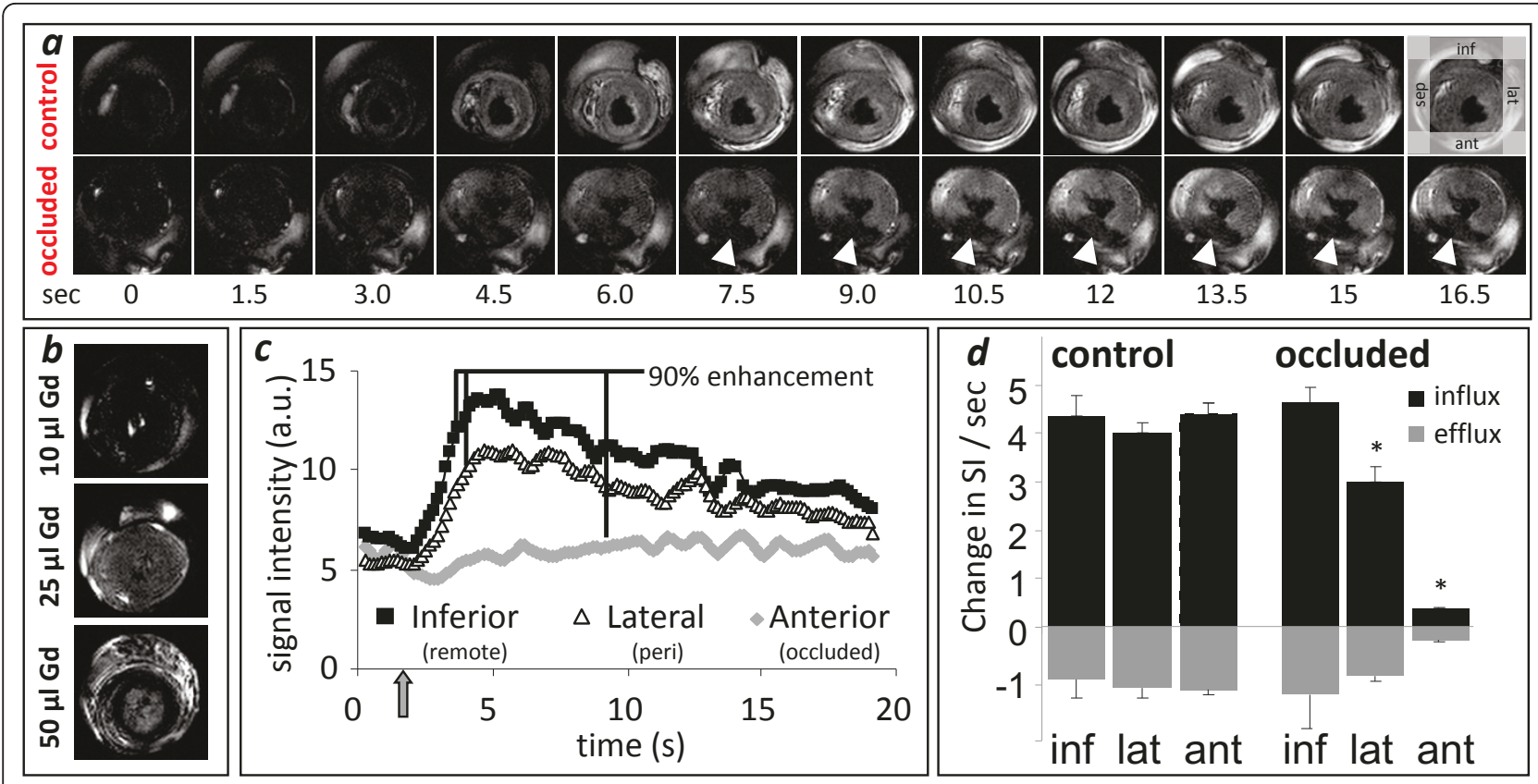

Figure 1 First-pass imaging of the isolated, perfused rat heart. (a) 12 of the 128 images acquired prior to, during and after bolus Gd infusion from control and ex vivo occluded perfused hearts: ( $\boldsymbol{b}$ ) Images showing effect of different Gd bolus volumes; (c) Signal intensity/time curves from inferior (inf), lateral (lat) and anterior (ant) regions of a heart in which the coronary artery was occluded on the rig. Arrow indicates time of bolus infusion: $(\boldsymbol{d})$ Graph of rates of change in signal intensity in different regions of control $(n=12)$ and ex vivo occluded ( $n=8)$ hearts. Positive values calculated from contrast influx, negative values calculated from contrast efflux. ${ }^{*} p<0.05$ compared with lateral remote tissue.

clearance of the Gd bolus from the myocardium was calculated as the gradient of the signal intensity/time curve from 10 to 15 seconds after bolus infusion. No differences in $\mathrm{Gd}$ efflux between regions were observed (Figure 1d).

Perfused hearts were then removed from the magnet and the LAD occluded (successful occlusion in 8 of 12 hearts). Areas of low perfusion were identified below the occluded LAD as non-enhanced regions on MR images acquired during the Gd bolus infusion (Figure 1a). Perfusion delay (PD), calculated as the delay between time to 90\% enhancement in an ROI compared with the remote inferior wall (Figure 1c), was significantly longer in the infarcted anterior wall $(3.47 \pm 0.65 \mathrm{~s}, \mathrm{p}<0.001)$ and the peri-infarcted lateral wall $(0.81 \pm 0.49 \mathrm{~s}, \mathrm{p}<0.05)$. The gradient of signal intensity increase was significantly lower in the infarcted $(0.40 \pm 0.02$ a.u./s, p < 0.05) and peri infarcted $(3.00 \pm 0.20$ a.u./s, $\mathrm{p}<0.05)$ regions compared with remote regions $(4.65 \pm 0.30$ a.u./s, Figure $1 \mathrm{~d})$. The rate of Gd clearance was also significantly lower in anterior myocardial regions affected by LAD occlusion (infarct, $-0.28 \pm 0.04$, peri-infarct $-1.00 \pm 0.20$, remote $-1.40 \pm 0.68$ a.u./s, Figure 1d). Saturated gradient echo images, acquired up to $50 \mathrm{~min}$ after coronary occlusion found no evidence of LGE in the perfused infarcted hearts.
In vivo first-pass perfusion imaging of infarcted rat hearts Cardiac morphology and function

Cine-MRI measurements of cardiac morphology and function were made serially on rats prior to and at 2, 8 and 42 days after infarction (Table 1). Left ventricular mass, end diastolic volume (EDV), end systolic volume (ESV) and body weight were significantly increased at 8 and 42 days after infarction. Ejection fraction (EF) was reduced by 2 days, whereas stroke volume and cardiac output were depressed at 2 days, but returned to normal levels by 8 days. The percentage of akinetic myocardium and heart rates did not change from 2 to 42 days.

First-pass cardiac perfusion imaging 2 and 8 days post infarction

Infusion of Gd initially reduced signal in the LV cavity owing to T1 and T2 shortening of blood from the high concentration of contrast agent (Figure 2a). This made it impossible to measure an arterial input function. However, signal in the myocardium became enhanced shortly after cavity hypoenhancement, as contrast agent perfused through the coronary circulation. Slight cyclical elevations in myocardial signal intensity occurred due to respiratory motion. Even though the image acquisition time continued through much of the cardiac cycle, cardiac motion did not prevent measurement of contrast inflow. Imaging of first-pass perfusion in rat hearts 
Table 1 In vivo measurements of rat heart morphology and function before and at 2, 8 and 42 days after infarction ( $=27$ )

\begin{tabular}{|c|c|c|c|c|}
\hline & Pre & 2 days & 8 days & 42 days \\
\hline Left ventricular mass (mg) & $492 \pm 35$ & $543 \pm 18^{*}$ & $571 \pm 15^{*}$ & $586 \pm 16^{* \#}$ \\
\hline End diastolic volume $(\mu \mathrm{l})$ & $265 \pm 12$ & $282 \pm 11$ & $395 \pm 15^{* \#}$ & $428 \pm 24^{* \#+}$ \\
\hline End systolic volume $(\mu \mathrm{l})$ & $55 \pm 8$ & $108 \pm 5^{*}$ & $154 \pm 13^{* \#}$ & $185 \pm 19^{* \#+}$ \\
\hline Stroke volume $(\mu \mathrm{l})$ & $210 \pm 9$ & $174 \pm 8^{*}$ & $241 \pm 7^{\#}$ & $243 \pm 11^{\#}$ \\
\hline Ejection fraction (\%) & $80 \pm 2$ & $62 \pm 1^{*}$ & $62 \pm 2^{*}$ & $58 \pm 2^{* \#+}$ \\
\hline Akinetic myocardium $\left(\mathrm{mm}^{2}\right)$ & - & $16 \pm 4^{*}$ & $23 \pm 5^{* \#}$ & $28 \pm 6^{* \#+}$ \\
\hline Akinetic myocardium (\%) & - & $9 \pm 2^{*}$ & $10 \pm 2^{*}$ & $11 \pm 2^{*}$ \\
\hline Heart rate (bpm) & $404 \pm 11$ & $361 \pm 9$ & $344 \pm 27$ & $346 \pm 26$ \\
\hline Cardiac output (ml/min) & $85 \pm 5$ & $61 \pm 3^{*}$ & $83 \pm 3^{\#}$ & $80 \pm 3^{\#}$ \\
\hline Weight (g) & $234 \pm 11$ & $214 \pm 3$ & $230 \pm 5$ & $245 \pm 19$ \\
\hline
\end{tabular}

${ }^{*} \mathrm{p}<0.05$ compared with pre-infarction. ${ }^{*} \mathrm{p}<0.05$ compared with 2 days. ${ }^{+} \mathrm{p}<0.05$ compared with 8 days,

identified areas of perfusion deficit in the region affected by the LAD coronary artery occlusion (Figure $2 \mathrm{a}, \mathrm{b}$ ). The average perfusion delay in infarct and peri-infarct compared with remote tissue was $4.0 \pm 0.7$ and $1.7 \pm$ $0.4 \mathrm{~s}$ at 2 days post infarction and $3.3 \pm 0.5$ and $0.5 \pm$ $0.3 \mathrm{~s}$ at 8 days post infarction.

\section{Late Gadolinium enhancement imaging 2 and 8 days post} infarction

LGE-CMR was performed 10-25 min after first-pass imaging. Hyperenhancement was present in $18 \pm 2 \%$ of the LV volume and $19 \pm 2 \%$ of LV endocardial perimeter at 2 days post infarction, and $11 \pm 1 \%$ of the LV volume and $16 \pm 2 \%$ of $\mathrm{LV}$ endocardial perimeter at 8 days post infarction (Figure 2c).

For the sub-group of infarcted rats $(n=14)$ in which tail vein canulation was successful at both time points, first-pass CMR and LGE-CMR were performed at both 2 and 8 days post infarction. Perfusion delay (measured using first-pass CMR) and LGE-CMR endocardial scar perimeter were unchanged from 2 to 8 days (Table 2).

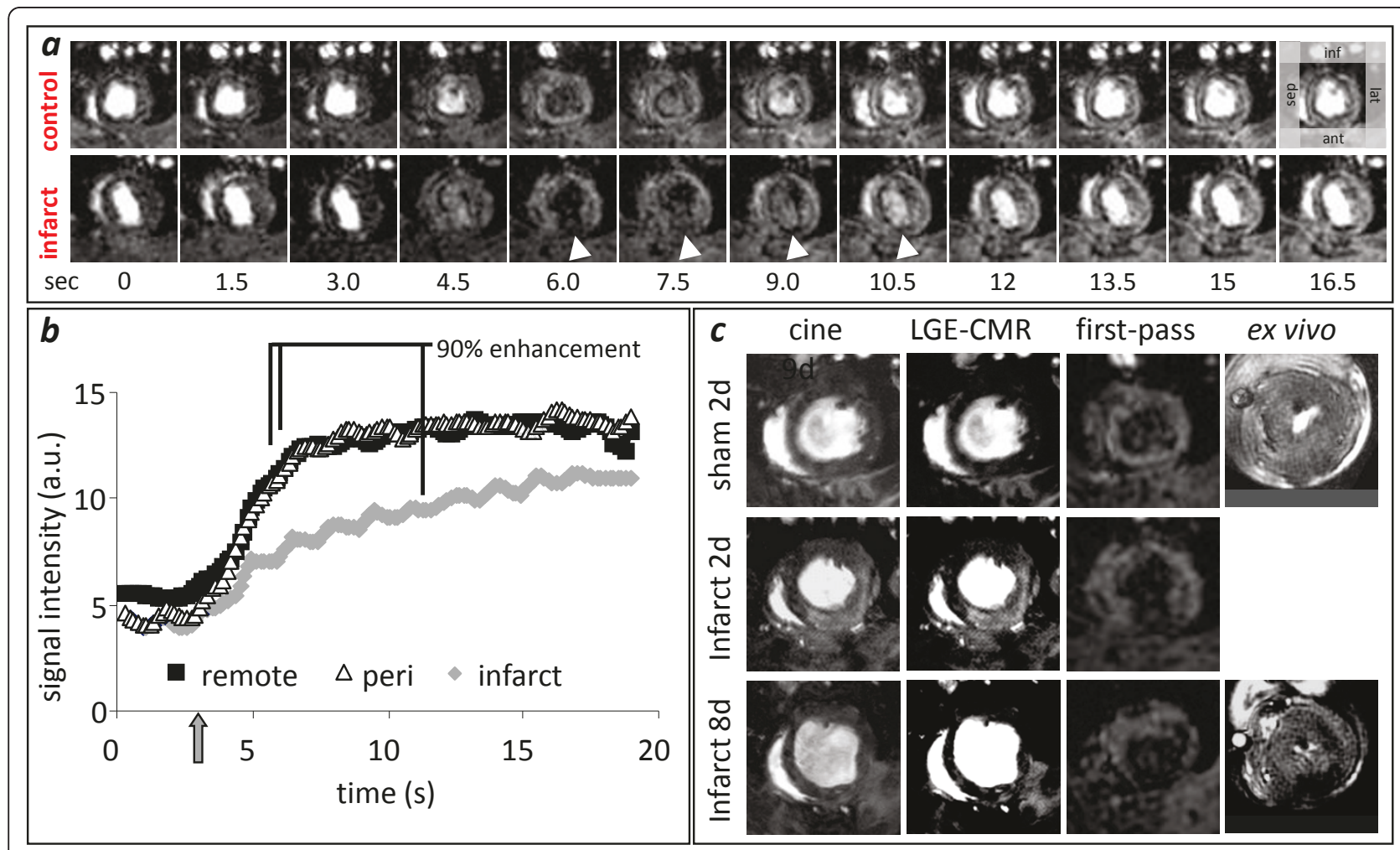

Figure 2 In vivo first-pass imaging. (a) 12 of the 128 images acquired prior to, during and after bolus Gd infusion from control and in vivo infarcted rat hearts: $(\boldsymbol{b})$ signal intensity/time curves from infarcted, peri-infarcted and remote regions of an infarcted heart. Arrow indicates time of bolus infusion: (c) in vivo cine-CMR, LGE-CMR and first-pass images from a sham operated rat and an infarcted rat at 2 and 8 days post surgery. First-pass images from the same isolated, perfused hearts are presented on the far right. 
Table 2 In vivo measurements of cardiac perfusion and viability in infarcted rat hearts $(n=14)$ at 2 and 8 days after infarction

\begin{tabular}{lcc}
\hline & 2 days & 8 days \\
\hline Perfusion delay $(s)$ & $3.57 \pm 0.71$ & $3.51 \pm 0.58$ \\
LGE-CMR \% perimeter & $18 \pm 3$ & $15 \pm 3$ \\
LGE-CMR \% volume & $19 \pm 3$ & $10 \pm 2^{\#}$ \\
LGE-CMR volume $(\mu l)$ & $100 \pm 18$ & $56 \pm 11^{\#}$ \\
\hline
\end{tabular}

$\#_{p}<0.01$ compared with 2 days.

However, there was a significant reduction in LGE-CMR scar size (measured as \% LV volume) between 2 and 8 days ( $19 \pm 3$ and $10 \pm 2 \%$ respectively, $p<0.01$; Figure 3 and Table 2) and in absolute scar size (100 \pm $18 \mu \mathrm{l}$ at 2 days vs. $56 \pm 11 \mu \mathrm{l}$ at 8 days, $p<0.01)$.

\section{Correlations between MRI measurements at 2 and 8 days} and ejection fractions and end systolic volumes at 42 days Perfusion delays measured at 2 days post infarction correlated with left ventricular end systolic volumes $(R=$ $0.84, P<0.0001)$ and ejection fractions $(R=0.85, P<$ 0.001 ) measured at 42 days (Figure 4 ), suggesting that rats with acute perfusion deficit went on to develop greater chronic cardiac impairment. As anticipated, infarct size measured using LGE-CMR at 2 days post infarction correlated with left ventricular end systolic volumes $(R=0.67, P<0.01)$ and ejection fractions $(R=$ $0.74, P<0.01$ ) measured at 42 days (Figure 4 ). However, correlations between infarct size and EF or ESV were lower than those found using first-pass CMR. At 2 days, perfusion delay gave a better prediction of outcome than cine MRI measurements of morphology and function, with no correlation between 2 day end systolic volumes and ejection fractions and the end systolic

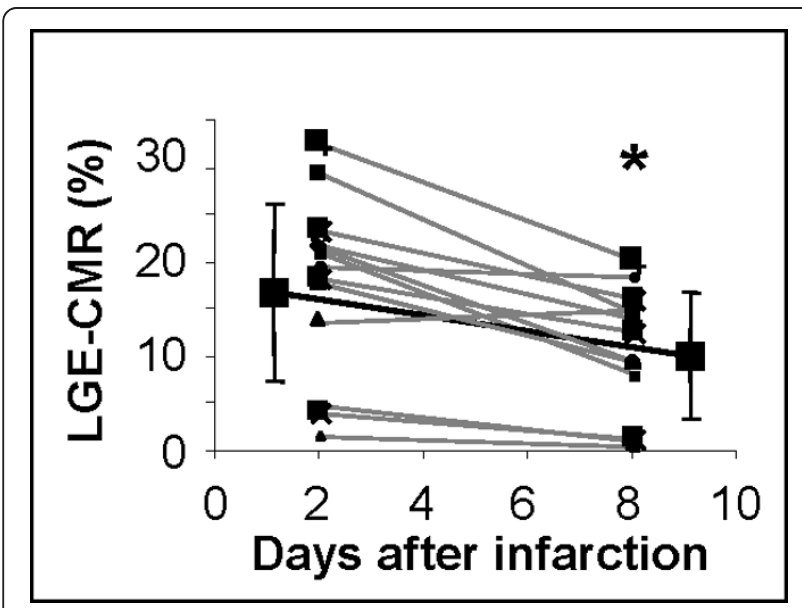

Figure 3 Repeated measures of LGE-CMR volume made in the same 14 infarcted rat hearts that had successful tail vein cannulation at 2 and 8 days after infarction. ${ }^{*} p<0.05$ compared with 2 day data. volumes and ejection fractions measured at 42 days. Perfusion delay measured at 8 days post infarction also correlated with $\operatorname{ESV}(R=0.76, P<0.05)$ and EF $(R=$ $0.63, P<0.05)$ at 42 days. Again, these correlations were stronger than those between LGE-CMR, ESV and EF at 8 days and the resulting ejection fractions and end systolic volumes measured at 42 days (data not shown).

First-pass cardiac perfusion imaging in isolated, perfused infarcted rat hearts

Nine days after infarction, 7 rat hearts were excised, perfused in Langendorff mode and imaged. Infusion of $25 \mu \mathrm{l} \mathrm{Gd}$ into the perfusate proximal to the aorta gave uniform signal enhancement throughout the remote myocardium, whereas infarcted regions showed only limited enhancement (Figure 2c). Signal intensity/time curves plotted from images acquired at the same location in vivo and ex vivo showed similar characteristics and resulted in similar measurements of perfusion delay (in vivo $=2.9 \pm 2.1 \mathrm{~s}$, ex vivo $=2.8 \pm 0.8 \mathrm{~s}$, correlation; $\left.R^{2}=0.7 . p<0.05\right)$. The gradient of signal intensity increase was significantly lower in the infarcted and peri infarcted regions compared with remote tissue (infarct, $0.38 \pm 0.03$, peri $2.36 \pm 0.42$, remote $4.31 \pm 0.91, \mathrm{p}<$ 0.01 ), while the rate of Gd clearance was significantly lower in infarcted regions compared with remote (infarct, $0.05 \pm 0.04$, peri $0.25 \pm 0.08$, remote $0.44 \pm$ $0.05)$. These experiments also indicated that the volume of reduced perfusion measured ex vivo matched the volume of LGE measured in vivo (Additional file 1). Interestingly, saturated gradient echo images performed 10 to 50 minutes after Gd infusion found no evidence of LGE in the perfused infarcted hearts. This probably occurred because Gd was infused as a single $25 \mu \mathrm{l}$ bolus and heart perfusate was not circulated, preventing accumulation of $\mathrm{Gd}$ in the infarcted tissue

\section{Discussion}

Clinical studies have demonstrated that the extent of myocardial perfusion deficit after infarction is a strong predictor of functional outcome, with patients who present with large microvascular obstructions (MVO) having poor prognosis [10]. MR imaging of the first-pass of a bolus infusion of $\mathrm{Gd}$ is now a well established technique for accurate identification and localisation of MVOs [5,11-13,30]. Despite its importance, first-pass MR imaging has only very recently been used in small animal models of myocardial infarction [24,25]. Here we developed a CMR method which acquired one image every heart cycle and was sensitive to the inflow of Gd into the myocardium, thus providing a rapid technique for assessing tissue perfusion.

Several methods have been used to assess myocardial perfusion in small animal models. Arterial spin labelling and $\mathrm{T}_{1}$ mapping can provide quantitative measurement 


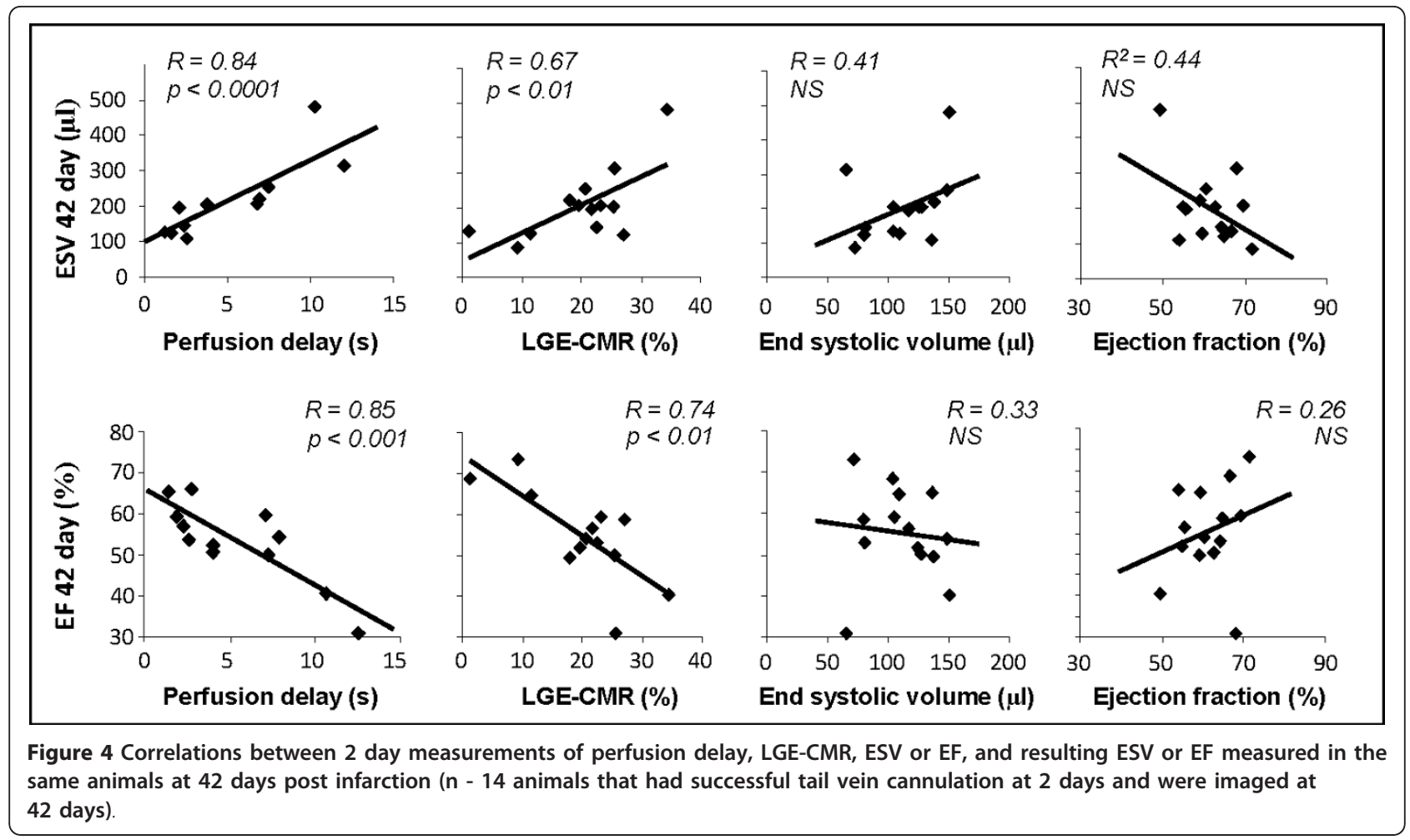

of blood flow post infarction in rats and mice [20-23]. However, the accuracy of these techniques is decreased by rapid and variable heart rates, artefacts produced by respiratory motion during acquisition and a single slice acquisition takes tens of minutes. PET imaging of radiotracers can be used to measure myocardial perfusion, but low spatial resolution, attenuation artefacts and the need for a local cyclotron to produce tracers, such as ${ }^{82} \mathrm{Rb}$ or $\mathrm{H}_{2}{ }^{15} \mathrm{O}$, limit the application of this technology, especially when applied to small animals [5,11-13,30,31].

We initially developed and optimised the first-pass method using isolated perfused rat hearts. This preparation permitted repeated contrast infusions in the same heart so that Gd bolus volume, concentration and infusion time could be optimised in a controlled environment. Repeated infusions indicated good reproducibility and uniform enhancement in anterior, lateral and inferior myocardial regions. To determine the sensitivity of the first-pass technique to regional perfusion deficits the LAD coronary artery was occluded and first-pass imaging was repeated. Signal intensity was measured in regions of interest placed in infarcted, peri-infarcted and remote tissue of all 128 images acquired during bolus infusion. Two methods were used to quantify regional perfusion changes. Firstly, perfusion delay was calculated as the time delay, in seconds, between $90 \% \max$ enhancement in infarcted regions compared with remote regions. Secondly, in ex vivo perfused hearts it was possible to assess contrast influx rate from the linear portion of the positive gradient of the signal intensity/ time curve and contrast efflux rate from the signal intensity/time curve 10-15 seconds after infusion. As both of these analysis methods relate changes in infarcted regions with those of remote regions of the same infusion any effects of slight differences in bolus transit time and volume can be reduced, providing more reproducible data.

The first-pass imaging method had sufficient $T_{1}$ weighting and temporal and spatial resolution to permit in vivo measurement of contrast inflow into the myocardium. The requirement for a rapid imaging sequence that could acquire an image every heart beat favoured a fast gradient echo acquisition rather than the more traditional inversion recovery sequence. The fast gradient echo acquisition resulted in signal saturation within the myocardium prior to contrast arrival, but did not null signal in the blood pool due to the rapid influx. Contrast transit through the ventricular cavities reduced signal intensity owing to the susceptibility effects of high $\mathrm{Gd}$ concentration at $11.7 \mathrm{~T}$. Importantly the Gd bolus resulted in signal enhancement within the perfused myocardial tissue. As with the isolated perfused heart experiments, signal intensity was measured in regions of interest placed in infarcted, peri-infarcted and remote tissue of all 128 images acquired during bolus infusion and perfusion delay was calculated as the time delay in 
seconds between $90 \%$ max enhancement in infarcted regions compared with remote regions. Perfusion delay in the infarcted regions was significantly longer than in remote tissue. Owing to the need for acquisition of high temporally resolved images, the in vivo spatial resolution of the first-pass technique is limited. Although regions of interest could be selected within the infarct territory, it was not possible to accurately assess the area of the perfusion deficit. This is augmented in the thinned scar tissue of the chronically infarcted heart and can make delineation of the endocardial border problematic. A further limitation of the method is that it cannot be performed at multiple slice positions during a single $\mathrm{Gd}$ bolus, while repeated bolus imaging is complicated by LGE in infarcted regions.

The severity of perfusion delay and extent of delayed enhancement measured at 2 and 8 days strongly correlated with cardiac function measured in the same rats 42 days after infarction. These correlations were stronger than those between ejection fraction and end systolic volume measured at 2 or 8 days and ejection fraction or end systolic volume at 42 days. Acutely after infarction, myocardial stunning, edema, and elevated catecholamine levels can strongly influence EF and ESV, masking the extent of systolic dysfunction. A combination of these factors, coupled with the variability of infarct size typically induced by experimental myocardial infarction, makes acute measurement of EF and ESV a poor early predictor of outcome and suggests that the common practice of dividing infarcted animals into experimental groups based on EF measured made principle by echocardiography in the first week post infarction can lead to wide variability in extent of functional impairment between groups. The first-pass and LGE-CMR techniques presented here gave better predictors of outcome cine-MRI, provided a more accurate assessment of myocardial viability acutely after infarction and may offer a useful baseline measure of infarct severity prior to administration of the therapeutic agent.

Ex vivo first-pass imaging was performed on isolated, perfused infarcted rat hearts that had undergone in vivo imaging at 2 and 8 days post infarction. In vivo and ex vivo measurements of perfusion delay correlated, as did in vivo LGE-CMR volume and ex vivo low perfusion volume and Gd clearance time. This supports the hypothesis that LGE-CMR is due to reduced washout and hence greater retention of $\mathrm{Gd}$ in infarcted regions [32].

The volume of LGE significantly decreased from 2 days to 8 days post infarction, and was unquantifiable at 42 days. Similar results were reported in canine models [33] and in two recent clinical studies which found a decrease in LGE from 12 to $8 \%$ of the left ventricle between 1 and 8 days after infarction [34] and from 27 to $22 \%$ between 24 hours to 6 months [35]. This decrease is probably due to hyperenhancement of oedematous, inflamed, viable tissue within the infarct border zone at 2 days post infarction, and thinning of the scar, infarct resorption and hypertrophy of remote tissue at 8 days [33,34]. However, it draws attention to possible limitations of using LGE-CMR in small animal models of chronic infarction where transmurality and tissue remodelling can make infarct assessment difficult. Few studies have used LGECMR chronically after infarction, and those that have did not study animals with large transmural infarcts [36], The first-pass CMR measurements of perfusion delays measured in this study did not change from 2 to 8 days post infarction, but we predict that the spatial resolution of the technique may be inadequate for measuring perfusion in established transmural scar tissue.

The resolution of the first-pass method may be improved through parallel imaging using multi channel phase array coils and accelerated image acquisitions. Schneider et al recently demonstrated the feasibility of using parallel imaging to achieve threefold acceleration of cine image acquisition in mice [37]. By using similar strategies, it may be possible to perform multi slice first-pass imaging with a spatial resolution that permits accurate delineation of underperfused areas of myocardium, or to assess changes in perfusion in peri-infarct areas in response to dobutamine stress or after experimental therapy with angiogenic cytokines or stem cells. A novel therapeutic strategy for replacing myocardium lost during infarction is grafting of engineered heart tissue $[38,39]$. It is currently difficult to assess in vivo whether the graft is incorporated into the host's vasculature. First-pass CMR may be able to detect contrast agent inflow into the engineered tissue and determine successful integration.

\section{Conclusions}

In summary, we have presented a novel, simple and useful MR method for identification of under-perfused tissue in the infarcted rat heart and demonstrate the high prognostic value of the technique compared with other non-invasive measures of cardiac function and viability. This method is a useful tool for assessing the severity of myocardial ischemia acutely after infarction and could provide important data on treatment strategies designed to revascularise and regenerate the infarcted heart.

\section{Additional material}

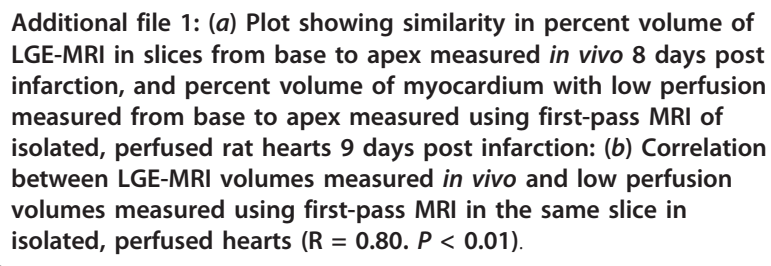

Additional file 1: (a) Plot showing similarity in percent volume of LGE-MRI in slices from base to apex measured in vivo 8 days post infarction, and percent volume of myocardium with low perfusion measured from base to apex measured using first-pass MRI of isolated, perfused rat hearts 9 days post infarction: $(b)$ Correlation between LGE-MRI volumes measured in vivo and low perfusion volumes measured using first-pass MRI in the same slice in isolated, perfused hearts $(\mathrm{R}=0.80 . P<0.01)$. 


\section{Abbreviations}

LGE-CMR: late gadolinium enhancement cardiac magnetic resonance; EF: ejection fraction; EDV: end diastolic volume; ESV: end systolic volume; FLASH: fast low angle shot; Gd-DTPA: gadolinium diethylenetriaminepentaacetate; LAD: left anterior descending coronary artery; LV: left ventricle; MVO: microvascular obstruction; PD: pefusion delay.

\section{Acknowledgements}

This work was supported by The British Heart Foundation (grant number PG/07/059/23259).

\section{Author details}

'Department of Physiology, Anatomy and Genetics, University of Oxford, Parks Road, Oxford, UK. ${ }^{2}$ Biological Imaging Centre, Imperial College, Hammersmith Hospital, Du Cane Road, London W12 ONN, UK.

\section{Authors' contributions}

DJS conceived and designed the study, performed all MRI and analysis, and drafted the manuscript; CAC performed all surgical procedures and assisted with MRl; SJM and MAC assisted with perfused heart experiments, DJT assisted with MRI; KC assisted with study design, manuscript preparation and funding. All authors read and approved the final manuscript

\section{Competing interests}

The authors declare that they have no competing interests.

Received: 21 February 2011 Accepted: 3 August 2011

Published: 3 August 2011

\section{References}

1. Saeed M: New concepts in characterization of ischemically injured myocardium by MRI. Exp Biol Med (Maywood) 2001, 226:367-376.

2. Schinkel AF, Poldermans D, Elhendy A, Bax JJ: Assessment of myocardial viability in patients with heart failure. J NuCl Med 2007, 48:1135-1146.

3. Wu KC, Lima JA: Noninvasive imaging of myocardial viability: current techniques and future developments. Circ Res 2003, 93:1146-1158.

4. Nijveldt R, Beek AM, Hirsch A, Stoel MG, Hofman MB, Umans VA, Algra PR, Twisk JW, van Rossum AC: Functional recovery after acute myocardial infarction: comparison between angiography, electrocardiography, and cardiovascular magnetic resonance measures of microvascular injury. J Am Coll Cardiol 2008, 52:181-189.

5. Plein S, Younger JF, Sparrow P, Ridgway JP, Ball SG, Greenwood JP: Cardiovascular magnetic resonance of scar and ischemia burden early after acute ST elevation and non-ST elevation myocardial infarction. I Cardiovasc Magn Reson 2008, 10:47.

6. Orn S, Manhenke C, Anand IS, Squire I, Nagel E, Edvardsen T, Dickstein K: Effect of left ventricular scar size, location, and transmurality on left ventricular remodeling with healed myocardial infarction. Am J Cardiol 2007, 99:1109-1114.

7. Ingkanisorn WP, Rhoads KL, Aletras AH, Kellman P, Arai AE: Gadolinium delayed enhancement cardiovascular magnetic resonance correlates with clinical measures of myocardial infarction. J Am Coll Cardiol 2004, 43:2253-2259.

8. Kim RJ, Wu E, Rafael A, Chen EL, Parker MA, Simonetti O, Klocke FJ, Bonow RO, Judd RM: The use of contrast-enhanced magnetic resonance imaging to identify reversible myocardial dysfunction. N Engl J Med 2000, 343:1445-1453.

9. Atkinson DJ, Burstein D, Edelman RR: First-pass cardiac perfusion: evaluation with ultrafast MR imaging. Radiology 1990, 174:757-762.

10. Gerber BL, Raman SV, Nayak K, Epstein FH, Ferreira P, Axel L, Kraitchman DL: Myocardial first-pass perfusion cardiovascular magnetic resonance: history, theory, and current state of the art. J Cardiovasc Magn Reson 2008, 10:18.

11. Lombardo A, Rizzello V, Galiuto L, Natale L, Giordano A, Rebuzzi A, Loperfido F, Crea F, Maseri A: Assessment of resting perfusion defects in patients with acute myocardial infarction: comparison of myocardial contrast echocardiography, combined first-pass/delayed contrastenhanced magnetic resonance imaging and 99mTC-sestamibi SPECT. Int J Cardiovasc Imaging 2006, 22:417-428.

12. Lund GK, Stork A, Saeed M, Bansmann MP, Gerken JH, Muller V, Mester J, Higgins $C B$, Adam G, Meinertz T: Acute myocardial infarction: evaluation with first-pass enhancement and delayed enhancement MR imaging compared with 201TI SPECT imaging. Radiology 2004, 232:49-57.
13. Nagel E, Klein C, Paetsch I, Hettwer S, Schnackenburg B, Wegscheider K, Fleck E: Magnetic resonance perfusion measurements for the noninvasive detection of coronary artery disease. Circulation 2003, 108:432-437.

14. Schwitter J, Nanz D, Kneifel S, Bertschinger K, Buchi M, Knusel PR, Marincek B, Luscher TF, von Schulthess GK: Assessment of myocardial perfusion in coronary artery disease by magnetic resonance: a comparison with positron emission tomography and coronary angiography. Circulation 2001, 103:2230-2235.

15. Epstein FH: MRI of left ventricular function. J Nucl Cardiol 2007, 14:729-744.

16. Pfeffer MA, Pfeffer JM, Steinberg C, Finn P: Survival after an experimental myocardial infarction: beneficial effects of long-term therapy with captopril. Circulation 1985, 72:406-412.

17. Nahrendorf M, Wiesmann F, Hiller KH, Hu K, Waller C, Ruff J, Lanz TE, Neubauer S, Haase A, Ertl G, Bauer WR: Serial cine-magnetic resonance imaging of left ventricular remodeling after myocardial infarction in rats. J Magn Reson Imaging 2001, 14:547-555.

18. Carr CA, Stuckey DJ, Tatton L, Tyler DJ, Hale SJ, Sweeney D, Schneider JE, Martin-Rendon E, Radda GK, Harding SE, et al: Bone marrow-derived stromal cells home to and remain in the infarcted rat heart but fail to improve function: an in vivo cine-MRI study. Am J Physiol Heart Circ Physiol 2008, 295:H533-542.

19. Bohl S, Lygate CA, Barnes H, Medway D, Stork LA, Schulz-Menger J, Neubauer S, Schneider JE: Advanced methods for quantification of infarct size in mice using three-dimensional high-field late gadolinium enhancement MRI. Am J Physiol Heart Circ Physiol 2009, 296:H1200-1208.

20. Kober F, lltis I, Cozzone PJ, Bernard M: Myocardial blood flow mapping in mice using high-resolution spin labeling magnetic resonance imaging: influence of ketamine/xylazine and isoflurane anesthesia. Magn Reson Med 2005, 53:601-606.

21. Nahrendorf M, Streif JU, Hiller KH, Hu K, Nordbeck P, Ritter O, Sosnovik DE, Bauer L, Neubauer S, Jakob PM, et al: Multimodal functional cardiac MR imaging in creatine kinase deficient mice reveals subtle abnormalities in myocardial perfusion and mechanics. Am J Physiol Heart Circ Physiol 2006.

22. Bauer WR, Hiller KH, Galuppo P, Neubauer S, Kopke J, Haase A, Waller C, Ertl G: Fast high-resolution magnetic resonance imaging demonstrates fractality of myocardial perfusion in microscopic dimensions. Circ Res 2001, 88:340-346.

23. Waller $C$, Engelhorn T, Hiller KH, Heusch G, Ertl G, Bauer WR, Schulz R: Impaired resting perfusion in viable myocardium distal to chronic coronary stenosis in rats. Am J Physiol Heart Circ Physiol 2005, 288: H2588-2593.

24. Makowski M, Jansen C, Webb I, Chiribiri A, Nagel E, Botnar R, Kozerke S, Plein S: First-pass contrast-enhanced myocardial perfusion MRI in mice on a 3-T clinical MR scanner. Magn Reson Med 2010, 64:1592-1598.

25. Coolen BF, Moonen RP, Paulis LE, Geelen T, Nicolay K, Strijkers GJ: Mouse myocardial first-pass perfusion MR imaging. Magn Reson Med 2010.

26. Stuckey DJ, Carr CA, Martin-Rendon E, Tyler DJ, Willmott C, Cassidy PJ, Hale SJ, Schneider JE, Tatton L, Harding SE, et al: Iron particles for noninvasive monitoring of bone marrow stromal cell engraftment into, and isolation of viable engrafted donor cells from, the heart. Stem Cells 2006, 24:1968-1975.

27. Cassidy PJ, Schneider JE, Grieve SM, Lygate C, Neubauer S, Clarke K: Assessment of motion gating strategies for mouse magnetic resonance at high magnetic fields. J Magn Reson Imaging 2004, 19:229-237.

28. Stuckey DJ, Carr CA, Tyler DJ, Aasum E, Clarke K: Novel MRI method to detect altered left ventricular ejection and filling patterns in rodent models of disease. Magn Reson Med 2008, 60:582-587.

29. Protti A, Sirker A, Shah AM, Botnar R: Late gadolinium enhancement of acute myocardial infarction in mice at 7T: cine-FLASH versus inversion recovery. J Magn Reson Imaging 2010, 32:878-886.

30. Heller GV, Calnon D, Dorbala S: Recent advances in cardiac PET and PET/ CT myocardial perfusion imaging. J Nucl Cardiol 2009, 16:962-969.

31. Schelbert HR, Inubushi M, Ross RS: PET imaging in small animals. J Nucl Cardiol 2003, 10:513-520.

32. Mahrholdt H, Wagner A, Judd RM, Sechtem U, Kim RJ: Delayed enhancement cardiovascular magnetic resonance assessment of nonischaemic cardiomyopathies. Eur Heart J 2005, 26:1461-1474.

33. Fieno DS, Hillenbrand HB, Rehwald WG, Harris KR, Decker RS, Parker MA, Klocke FJ, Kim RJ, Judd RM: Infarct resorption, compensatory hypertrophy, 
and differing patterns of ventricular remodeling following myocardial infarctions of varying size. J Am Coll Cardiol 2004, 43:2124-2131.

34. Engblom H, Hedstrom E, Heiberg E, Wagner GS, Pahlm O, Arheden H: Rapid initial reduction of hyperenhanced myocardium after reperfused first myocardial infarction suggests recovery of the peri-infarction zone: one-year follow-up by MRI. Circ Cardiovasc Imaging 2009, 2:47-55.

35. Dall'armellina E, Karia N, Lindsay AC, Karamitsos TD, Ferreira V, Robson MD, Kellman P, Francis JM, Forfar C, Prendergast BD, et al: Dynamic changes of edema and late gadolinium enhancement after acute myocardial infarction and their relationship to functional recovery and salvage index. Circ Cardiovasc Imaging 2011, 4:228-236.

36. Gnyawali SC, Roy S, McCoy M, Biswas S, Sen CK: Remodeling of the ischemia-reperfused murine heart: 11.7-T cardiac magnetic resonance imaging of contrast-enhanced infarct patches and transmurality. Antioxid Redox Signal 2009, 11:1829-1839.

37. Schneider JE, Lanz T, Barnes H, Stork LA, Bohl S, Lygate CA, Ordidge RJ, Neubauer S: Accelerated cardiac magnetic resonance imaging in the mouse using an eight-channel array at 9.4 Tesla. Magn Reson Med 2011, 65:60-70.

38. Stuckey DJ, Ishii H, Chen QZ, Boccaccini AR, Hansen U, Carr CA, Roether JA, Jawad H, Tyler DJ, Ali NN, et al: Magnetic resonance imaging evaluation of remodeling by cardiac elastomeric tissue scaffold biomaterials in a rat model of myocardial infarction. Tissue Eng Part A 2010, 16:3395-3402.

39. Zimmermann WH, Melnychenko I, Wasmeier G, Didie M, Naito H, Nixdorff U, Hess A, Budinsky L, Brune K, Michaelis B, et al: Engineered heart tissue grafts improve systolic and diastolic function in infarcted rat hearts. Nat Med 2006, 12:452-458.

doi:10.1186/1532-429X-13-38

Cite this article as: Stuckey et al.: First-pass perfusion CMR two days after infarction predicts severity of functional impairment six weeks later in the rat heart. Journal of Cardiovascular Magnetic Resonance 2011 13:38.

\section{Submit your next manuscript to BioMed Central and take full advantage of:}

- Convenient online submission

- Thorough peer review

- No space constraints or color figure charges

- Immediate publication on acceptance

- Inclusion in PubMed, CAS, Scopus and Google Scholar

- Research which is freely available for redistribution

Submit your manuscript at www.biomedcentral.com/submit 